\title{
OSCILLATION AND NONOSCILLATION OF SOLUTIONS OF SECOND ORDER LINEAR DIFFERENTIAL EQUATIONS WITH INTEGRABLE COEFFICIENTS $\left(^{1}\right)$
}

\author{
BY
}

JAMES S. W. WONG

1. Introduction. We are here concerned with the oscillatory behavior of solutions of the following second order ordinary differential equation:

$$
x^{\prime \prime}+a(t) x=0, \quad t \geqq 0,
$$

where $a(t)$ is a locally integrable function of $t$. We call equation (1) oscillatory if all solutions of (1) have arbitrarily large zeros on $[0, \infty)$, otherwise, we say equation (1) is nonoscillatory. As a consequence of Sturm's Separation Theorem [21], if one of the solutions of (1) is oscillatory, then all of them are. The same is true for the nonoscillation of (1).

The literature on second order linear oscillation is voluminous. The first such result is of course the classical theorem of Sturm which asserts that

$$
a(t) \geqq a_{0}>0 \Rightarrow \text { oscillation, }
$$

and

$$
a(t) \leqq 0 \Rightarrow \text { nonoscillation, }
$$

where the inequalities in (2) and (3) are to be valid to all large $t\left({ }^{2}\right)$. Using the classical Euler's equation as the basic comparison equation:

$$
x^{\prime \prime}+\left(1 / 4 t^{2}\right) x=0
$$

Kneser [9] showed that

$$
t^{2} a(t) \leqq \frac{1}{4} \Rightarrow \text { nonoscillation, }
$$

and

$$
t^{2} a(t) \geqq(1+\varepsilon) / 4 \Rightarrow \text { oscillation. }
$$

Fite [5] was first to give an integral criteria for oscillation, namely

$$
a(t) \geqq 0 \text { and } \int^{\infty} a(t) d t=\infty \Rightarrow \text { oscillation. }
$$

Received by the editors October 25, 1968.

(1) Portions of this paper were based upon an address delivered to the Summer meeting of the Canadian Mathematical Congress on June 13, 1968, under the title, "Recent advances in second order oscillation theory".

$\left.{ }^{2}\right)$ We shall assume such an understanding throughout all our later discussions. 
Condition (7) was complemented by results of Hille [8] which states that if

$$
a(t) \geqq 0 \text { and } \int^{\infty} a(t) d t<\infty,
$$

then

$$
t A(t) \leqq \frac{1}{4} \Rightarrow \text { nonoscillation, }
$$

and

$$
t A(t) \geqq(1+\varepsilon) / 4 \Rightarrow \text { oscillation, }
$$

where

$$
A(t)=\int_{t}^{\infty} a(s) d s .
$$

Later, Wintner $[22]\left({ }^{3}\right)$ showed that Fite's condition remains valid without the additional assumption that $a(t)$ be nonnegative, i.e.

$$
\lim _{T \rightarrow \infty} \int_{0}^{T} a(s) d s=\infty \Rightarrow \text { oscillation. }
$$

Wintner [23] also proved under the assumption that

$$
\lim _{T \rightarrow \infty} \int_{0}^{T} a(s) d s<\infty,
$$

the following theorems:

$$
\int_{0}^{\infty} \exp \left(-k \int_{0}^{t} A(s) d s\right) d t<\infty, \quad k=2 \Rightarrow \text { oscillation }
$$

and

$$
A^{2}(t) \leqq a(t) / 4 \Rightarrow \text { nonoscillation. }
$$

Assertion (14) remains valid with $k=4$. This improvement was due to Hartman [6]. On the other hand, Opial [16] extended (15) to

$$
\int_{t}^{\infty} A^{2}(s) d s \leqq A(t) / 4 \Rightarrow \text { nonoscillation, }
$$

and complemented (16) by

$$
\int_{t}^{\infty} A^{2}(s) d s \geqq((1+\varepsilon) / 4) A(t) \geqq 0 \Rightarrow \text { oscillation. }
$$

The significance of integral conditions, e.g. (7), (12), (14) lies in the fact that no individual $t$-values occur explicitly in the condition. In other words, $a(t)$ and its repeated indefinite integral $A(t)$ can be of "arbitrarily irregular growth". However,

(3) Wintner actually proved a stronger assumption than that of (12), cf. Remark 2. 
the integral conditions are merely criteria for oscillation and gives no information concerning nonoscillation when the conditions fail to hold. On the other hand, the integral comparison tests of Kneser (5), (6), Hille (9), (10), and Opial (16), (17) are "within $\varepsilon$ " necessary and sufficient conditions for oscillation or nonoscillation of equation (1).

We are primarily interested in equations with integrable coefficients, namely those $a(t)$ satisfying (13). In this case, $A(t)$ exists for all $t$. If $A(t) \geqq 0$ for large $t$, then Opial's results, i.e. (16), (17), are applicable. However, for most interesting examples with $a(t)$ assuming negative values for large $t$, its iterated integral $A(t)$ will also assume negative values for large $t$, e.g. $a(t)=(\sin \beta t) / t, \beta \neq 0$. There seems to exist only a few results which cover equations with coefficients of this sort. Earlier results by Wintner [23], Hartman [6], and Moore [14], Opial [16], [17], and also comparison theorems due to Wintner [24] and Hartman [7, Exercise 7.9, p. 369] are applicable to certain situations. Recently, a few other papers appear which also treat this problem, e.g. Macki and Wong [11], Willett [25], and Coles and Willett [4]. However, a thorough study of the relationship between the integrability of the coefficients $a(t)$, (its iterated integral $A(t)$ ), and the oscillation of equation (1) has not been made. The purpose of this paper is to provide some ground work for generating oscillation criteria for equation (1) with integrable coefficients.

Consider the special example $a(t)=(\sin \beta t) / t$. Results of Willett [25] show that for this special example, equation (1) is oscillatory $\left({ }^{4}\right)$ if $|\beta|>1 / 2^{1 / 2}$ and nonoscillatory if $|\beta|<1 / 2^{1 / 2}$. Similar to Opial's extension (16) of Wintner's result (15), Willett obtained a further extension of Opial's result by integrating conditions (16), (17) in some appropriate manner. Our results here will show that this special equation is nonoscillatory when $|\beta|=1 / 2^{1 / 2}$. Moreover, our results are applicable to further perturbations of the boundary situation $|\beta|=1 / 2^{1 / 2}$, reminiscent of the RiemannWeber [18] extension of the Euler's equation:

$$
x^{\prime \prime}+\left(1 / 4 t^{2}+c / 4(t \log t)^{2}\right) x=0,
$$

which states that (18) is oscillatory if $c>\frac{1}{4}$ and nonoscillatory otherwise.

The basis for our extensions is the introduction of a new Riccati integral equation which is useful in this study especially which $A(t)$ is not nonnegative for large $t$. It will be shown that under suitable assumptions, one may iterate this new Riccati integral equation again and obtain another one of the same type. In $\S 2$, we establish the equivalence of nonoscillation of (1) and the existence of a solution of certain Riccati integral equation. In $\$ 3$, we prove generalizations of results of Opial [1] and Willett [25]. In $\$ 4$, we present some refinements of comparison theorems due

( $\left.{ }^{4}\right)$ The fact that $|\beta|=1 / 2^{1 / 2}$ is the dividing line for oscillation and nonoscillation of this special example was previously known to the author and J. J. Mahony by methods of asymptotic expansions. In fact, our "computational" technique is applicable to a large number of special examples of this sort. 
to Wintner [24] and Hartman [7]. We devote $\$ 5$ to a discussion of some relevant examples, most of which are so far not covered by the existing results. Finally, we indicate in $\$ 6$ how transformation of variables may be used to extend our results to a larger class of equations than those of equation (1). Portions of $\$ \S 2$ and 3 were announced earlier in Wong [26].

2. Basic theory. For later discussions, it is convenient to introduce the notion of disconjugacy. We say a solution $x(t)$ of (1) is disconjugate on the interval $\left[t_{0}, \infty\right)$, where $t_{0} \geqq 0$, if $x(t)$ has at most one zero in [ $\left.t_{0}, \infty\right)$. It is clear from Sturm's Separation Theorem that (1) is nonoscillatory if and only if it has a solution $x(t)$ which is disconjugate on $\left[t_{0}, \infty\right)$ for some $t_{0}$. The following lemma is basic for all later discussions and was due to Wintner [23], [24], the proof may be found in Hartman [7] and is hence omitted.

LEMma. The following statements are equivalent:

(i) Equation (1) is nonoscillatory.

(ii) There exists a function $\varphi \in C^{1}\left[t_{0}, \infty\right)$, where $t_{0}$ is some positive real number such that

$$
\varphi^{\prime}(t)+\varphi^{2}(t)+a(t) \leqq 0 .
$$

(iii) There exists a solution to the following Riccati integral equation:

$$
v(t)=A(t)+\int_{t}^{\infty} v^{2}(s) d s .
$$

We are now ready to prove the following three theorems which form the basis of the present theory.

THEOREM 1. The nonoscillation of (1) is equivalent to the existence of a solution to the following integral equation

$$
u(t)=\bar{A}(t)+\int_{t}^{\infty} Q_{A}(s, t) u^{2}(s) d s,
$$

where

$$
\bar{A}(t)=\int_{t}^{\infty} A^{2}(s) Q_{A}(s, t) d s,
$$

and

$$
Q_{A}(s, t)=\exp \left(2 \int_{t}^{s} A(\tau) d \tau\right)
$$

Proof. Assume that (21) has a solution $u(t)$, then an easy computation shows that $v(t)=u(t)+A(t)$ defines a solution of (20). As a consequence of the above lemma, we conclude that (1) is nonoscillatory. Conversely, if (1) is nonoscillatory then equation (20) has a solution $v(t)$. Define $u(t)=\int_{t}^{\infty} v^{2}(s) d s$. One easily sees that $u(t)$ satisfies the differential equation:

$$
u^{\prime}(t)=-(u(t)+A(t))^{2} .
$$


Using $Q_{A}(t, 0)$ as an integrating factor, we integrate (24) and obtain

$$
u(t)=u(T) Q_{A}(T, t)+\int_{t}^{T} A^{2}(s) Q_{A}(s, t) d s+\int_{t}^{T} u^{2}(s) Q_{A}(s, t) d s .
$$

Since all integrals on the right hand side of (25) have nonnegative integrands, we infer from (25) that $\bar{A}(t)$ exists and

$$
\lim _{T \rightarrow \infty} u(T) Q_{A}(T, t)=L(t)
$$

We now claim that the limit $L(t)$ in (26) must be zero for all $t$. Suppose that $L(t)>0$ for some $t$. Choose $t_{1} \geqq t$ such that for $s \geqq t_{1}$,

$$
u(s) Q_{A}(s, t) \geqq \frac{1}{2} L(t)>0 .
$$

Substituting (27) into the last integral in (25), one finds

$$
u(t) \geqq \int_{t}^{T} u^{2}(s) Q_{A}(s, t) d s \geqq \frac{1}{4}(L(t))^{2} \int_{t}^{T} Q_{A}(t, s) d s, \quad t \geqq t_{1} .
$$

Letting $T$ tend to infinity in (28), we obtain (14) which implies that (1) is oscillatory, which is a desired contradiction. Since $t$ is arbitrary, this shows that $L(t) \equiv 0$. Now we may replace $T$ in (25) by $\infty$ and readily obtain (21).

Under the assumption that $a(t)$ is integrable, Theorem 1 is a further refinement of the results of Hille [8] and Wintner [23] proving the equivalence between (i) and (iii) as stated in the above lemma. We remark that the integral Riccati equation (21) was also used by Willett [25] in obtaining oscillation and nonoscillation criteria to be further detailed in the next section. In fact, it was proved in [25] by entirely different methods that the nonexistence of a solution to the following integral Riccati inequality:

$$
u(t) \geqq \bar{A}(t)+\int_{t}^{\infty} Q_{A}(s, t) u^{2}(s) d s
$$

is sufficient for the oscillation of (1).

Using (21) instead of (20), we obtain the following results which complement the result of Hartman [6] in case $A(t)$ is integrable.

TheORem 2. If $A(t)$ is integrable, i.e.

$$
\lim _{T \rightarrow \infty} \int_{0}^{T} A(s) d s<\infty
$$

and $\bar{A}(t)$ satisfies

$$
\int_{0}^{\infty} \exp \left(-4 \int_{0}^{t} \bar{A}(u) d u\right) d t<\infty
$$

then (1) is oscillatory. 
Proof. Assume the contrary that (1) has a nonoscillatory solution $x(t)$. Define $v(t)=x^{\prime}(t) / x(t)$, then it follows from Theorem 1 that the function $u(t)=\int_{t}^{\infty} v^{2}(s) d s$ satisfies (21). Define $\rho(t)=\int_{t}^{\infty} u^{2}(s) Q_{A}(s, t) d s$, then one easily sees that $\rho$ satisfies

$$
\begin{aligned}
\rho^{\prime}(t) & =-u^{2}(t)-2 A(t) \rho(t) \\
& =-\rho^{2}(t)-2(\bar{A}(t)+A(t)) \rho(t)-\bar{A}^{2}(t) \\
& \leqq-(4 \bar{A}(t)+2 A(t)) \rho(t) .
\end{aligned}
$$

Using (29), (30) in (31), we obtain that $\int_{0}^{\infty} \rho(t) d t<\infty$. Observe that

$$
\begin{aligned}
\int_{t_{0}}^{T} \int_{t}^{\infty} u^{2}(s) Q_{A}(s, t) d s d t & \geqq \int_{t_{0}}^{T}\left(\int_{t_{0}}^{s} \exp \left(-2 \int_{0}^{t} A(\sigma) d \sigma\right) d t\right) u^{2}(s) Q_{A}(s, 0) d s \\
& \geqq c_{1} \int_{t_{0}}^{T} s u^{2}(s) Q_{A}(s, 0) d s, \quad c_{1}>0, \\
\left(\int_{t_{0}}^{T} u(s) d s\right)^{2} & \leqq\left(\int_{t_{0}}^{T} s u^{2}(s) Q_{A}(s, 0) d s\right)\left(\int_{t_{0}}^{T} \frac{1}{s} Q_{A}(0, s) d s\right),
\end{aligned}
$$

and

$$
\left(\int_{t_{0}}^{T} \frac{1}{s} Q_{A}(0, s) d s\right)^{2} \leqq\left(\int_{t_{0}}^{T} \exp \left(-4 \int_{0}^{s} A(\sigma) d \sigma\right) d s\right) \int_{t_{0}}^{T} \frac{d s}{s^{2}} \leqq c_{2} T .
$$

Using (32) and (34) in (33), we obtain that $\int_{0}^{T} u(s) d s \leqq c_{4} T^{1 / 2}$. From the definitions of $u(t)$ and $v(t)$, we have

$$
\left(\int_{t_{0}}^{T} v(s) d s\right)^{2} \leqq\left(\int_{t_{0}}^{T} s v^{2}(s) d s\right) \int_{t_{0}}^{T} \frac{d s}{s}
$$

and

$$
\left(\int_{t_{0}}^{T} s v^{2}(s) d s\right) \leqq \frac{1}{2} \int_{t_{0}}^{T} u(s) d s
$$

Using (35) and (36), we obtain the following estimate for the nonoscillatory solution $x(t)$, namely

$$
\log \left(\frac{x(t)}{x\left(t_{0}\right)}\right)=\int_{t_{0}}^{t} v(s) d s \leqq c_{5}(\log t)^{3 / 4}, \quad c_{5}>0,
$$

where $c_{5}$ as the previous $c_{i}$ 's is an appropriate positive constant depending only on $t_{0}$. As a consequence of (37), we have

$$
\frac{1}{x^{2}(t)} \geqq c_{6} \exp \left(-2 c_{5}(\log t)^{3 / 4}\right) \geqq c_{7} \exp \left(-\frac{1}{2} \log t\right), \quad c_{6}, c_{7}>0
$$

thus

$$
\int^{\infty} \frac{d t}{x^{2}(t)} \geqq c_{7} \int^{\infty} \exp \left(-\frac{1}{2} \log t\right) d t=\infty \text {. }
$$

This contradicts the existence of nonprincipal solutions (Hartman [7], p. 355) and completes the proof. 
We remark that the above proof is a modification of Hartman's proof of assertion (14) with $k=4$. Here, we apply the technique to equation (21) instead of (20). Now, we may use Theorem 2 in place of (14) to obtain a further Riccati integral equation in case $A(t)$ is integrable. The analogue of Theorem 1 is the following:

THEOREM 3. If $A(t)$ is integrable then (1) is nonoscillatory if and only if there exists a solution to the following integral equation:

$$
w(t)=\tilde{A}(t)+\int_{t}^{\infty} Q_{\bar{A}}(s, t) w^{2}(s) d s,
$$

where

$$
\tilde{A}(t)=\int_{t}^{\infty} \bar{A}^{2}(s) Q_{A}(s, t) d s
$$

and

$$
Q_{\bar{A}}(s, t)=\exp \left(2 \int_{t}^{s}(A(\tau)+\bar{A}(\tau)) d \tau\right)
$$

Proof. Assume that (38) has a solution $w(t)$, then an easy computation shows that $v(t)=w(t)+A(t)+\bar{A}(t)$ is a solution of (20), hence by the above Lemma, (1) is nonoscillatory. Conversely, if (1) is nonoscillatory then by Theorem 1, equation (21) has a solution $u(t)$. Define $w(t)=\int_{t}^{\infty} Q_{A}(s, t) u^{2}(s) d s$. It is easy to see that $w(t)$ satisfies

$$
w^{\prime}(t)=-w^{2}(t)-\bar{A}^{2}(t)-2(A(t)+\bar{A}(t)) w(t) .
$$

Using $Q_{\bar{A}}(t, 0)$ as an integrating factor, we can integrate (41) as before and obtain

$$
w(t)=w(T) Q_{\bar{A}}(T, t)+\int_{t}^{T} \bar{A}^{2}(s) Q_{\bar{A}}(s, t) d s+\int_{t}^{T} w^{2}(s) Q_{\bar{A}}(s, t) d s .
$$

Proceeding as in the proof of Theorem 1, we need to show that

$$
\lim _{T \rightarrow \infty} w(T) Q_{\bar{A}}(T, t)=\bar{C}(t) \equiv 0,
$$

for all large $t$. Suppose that $\bar{C}(\bar{t})>0$ for some $\bar{t}$. Choose $t_{2} \geqq \bar{t}$ such that for $s \geqq t_{2}$, we have

$$
w(s) Q_{\bar{A}}(s, \bar{t}) \geqq \frac{1}{2} \bar{C}(\bar{t})>0 .
$$

Substituting (43) into the last integral in (42), we obtain

$$
w(t) \geqq \int_{t}^{T} w^{2}(s) Q_{\bar{A}}(s, t) d s \geqq \frac{1}{4}(\bar{C}(\bar{t}))^{2} \int_{t}^{T} Q_{\bar{A}}(t, s) d s,
$$

for all $t \geqq t_{2}$. Letting $T \rightarrow \infty$ in (44), we obtain (30), which by Theorem 2 contradicts the assumption that (1) is nonoscillatory. This completes the proof.

It is clear from the proofs of Theorems 1, 2, 3 that the process of generating higher-order iterated Riccati integral equations may be continued if we assume 
further that the iterated integrals $A(t), \bar{A}(t), \ldots$ are integrable. As immediate consequences of Theorems 1 and 3 , we obtain the following oscillation criteria which seems to be new $\left({ }^{5}\right)$.

COROllary 1. (i) If $a(t)$ is integrable, then

$$
\int_{0}^{\infty} A^{2}(s) \exp \left(2 \int_{0}^{s} A(\tau) d \tau\right) d s=\infty \Rightarrow \text { oscillation }
$$

and (ii) if $A(t)$ is integrable then

$$
\int_{0}^{\infty} \bar{A}^{2}(s) \exp \left(2 \int_{0}^{s} \bar{A}(\tau) d \tau\right) d s=\infty \Rightarrow \text { oscillation }
$$

EXAMPLE 1. As a nontrivial example, one may take $a(t)=(\alpha \sin \beta t) /(\ln t)^{\lambda}$ which satisfies (45) for all $\alpha, \beta \neq 0$ and $\lambda>0$. Previous result implies oscillation only for $\lambda<1$, (cf. Macki and Wong [11]).

ExAMPLE 2. Consider $a(t)=(\alpha \sin \beta t) / t^{\nu}$. It is easy to check that in this case for all $\alpha$ and $\beta \neq 0(45)$ is satisfied if $0<\gamma<\frac{1}{2}$ but (46) is satisfied if $0<\gamma<1$. No previous result in literature, with the exception of Willett [25], seems to cover this example.

In the following sections, we shall develop other oscillation and nonoscillation criteria when (45) and (46) fail to hold.

3. Main results. In this section, we shall improve the results of Opial [16] and Willett [25] mentioned in the introduction, based upon our results in $\$ 2$. The following theorem is a further elaboration of Opial's condition (16).

THEOREM 4. If there exists a function $B(t)$ such that

$$
\operatorname{Max}\left(|A(t)|,\left|A(t)+\int_{t}^{\infty}(A(s)+B(s))^{2} d s\right|\right) \leqq|A(t)+B(t)|,
$$

then (1) is nonoscillatory.

Proof. We shall use condition (47) to prove the existence of a solution of (20) by the Caratheodory's successive approximation technique. Let $N$ be a positive integer. We define the sequence of functions $\left\{v_{N}(t)\right\}$ as follows:

$$
\begin{aligned}
v_{N}(t) & =A(t)+B(t), & & t \geqq N \\
& =A(t)+\int_{t+1 / N}^{\infty} v_{N}^{2}(s) d s, & & 0 \leqq t \leqq N .
\end{aligned}
$$

Equation (48) defines $v_{N}(t)$ successively on the intervals $[N-k / N, N-(k-1) / N]$ for $k=1,2, \ldots$, and hence $v_{N}(t)$ is defined on $[0, \infty)$. For $N-1 / N \leqq t<\infty$, we have from (48) that

$$
v_{N}(t) \leqq\left|A(t)+\int_{t}^{\infty}(A(s)+B(s))^{2} d s\right| \leqq|A(t)+B(t)|
$$

(5) The referee has kindly pointed out that Corollary 1(i) may also be derived from Theorem 1.2 of [25]. 
and

$$
v_{N}(t) \geqq A(t) \geqq-|A(t)| \geqq-|A(t)+B(t)| .
$$

Using (49) and (50) and a simple induction, it is easy to show that for all $t \geqq 0$, we have $\left|v_{N}(t)\right| \leqq|A(t)+B(t)| \leqq M$. We now wish to show that $\left\{v_{N}(t)\right\}$ forms an equi-oscillatory family of uniformly bounded functions on $[0, \infty)$, i.e. for each $\varepsilon>0$, there exist $\delta>0, N_{0}>0$ such that for $|s-t|<\delta$, we must have $\left|v_{N}(t)-v_{N}(s)\right|<\varepsilon$ for all $N>N_{0}$. Since $A(t) \rightarrow 0$, for each $\varepsilon>0$, we may choose $T \geqq 0$ such that for all $t \geqq T$, we have $|A(t)| \leqq \varepsilon / 4$. On the finite interval $[0, T], A(t)$ is continuous and hence uniformly continuous, therefore, there must exist $\delta_{1}>0$ such that for all $0 \leqq s \leqq t \leqq T,|A(s)-A(t)|<\varepsilon / 4$ whenever $|s-t|<\delta_{1}$. From (48), we may estimate as follows:

$$
\left|v_{N}(s)-v_{N}(t)\right| \leqq|A(s)-A(t)|+\int_{s+1 / N}^{t+1 / N} v_{N}^{2}(s) d s \leqq|A(s)-A(t)|+M|s-t| .
$$

Choose $\delta=\operatorname{Min}\left(\delta_{1}, \varepsilon / 2 M\right)$, one readily sees that $\left|v_{N}(s)-v_{N}(t)\right|<\varepsilon$, whenever $|s-t|<\delta, N \geqq N_{0}$. Now an application of Arzela-Ascoli's Theorem shows that $\left\{v_{N}(t)\right\}$ contains a pointwise convergent subsequence $\left\{v_{N_{k}}(t)\right\}$, which converges uniformly on every compact subinterval of $[0, \infty)$. Denote its pointwise limit by $\tilde{v}(t)$. Note that condition (47) implies there exists a sequence $\left\{t_{j}\right\}, t \rightarrow \infty$ as $j \rightarrow \infty$ such that $\left|A\left(t_{j}\right)+B\left(t_{j}\right)\right| \rightarrow 0$. Write equation (48) in the form

$$
v_{N_{k}}(t)=\int_{t}^{t_{j}} a(s) d s+\int_{t+1 / N_{k}}^{t_{j}} v_{N_{k}}^{2}(s) d s+\beta_{k}\left(t_{j}\right)
$$

where

$$
\beta_{k}\left(t_{j}\right)=A\left(t_{j}\right)+\int_{t_{j}}^{\infty} v_{N_{k}}^{2}(s) d s .
$$

From (47) it follows that $\left|\beta_{k}\left(t_{j}\right)\right| \leqq\left|A\left(t_{j}\right)+B\left(t_{j}\right)\right|$. Letting $k \rightarrow \infty$ in (51), we have

$$
\liminf _{k \rightarrow \infty} \beta_{k}\left(t_{j}\right) \leqq \tilde{v}(t)-\int_{t}^{t_{j}} a(s) d s+\int_{t}^{t_{j}} v^{2}(s) d s \leqq \limsup _{k \rightarrow \infty} \beta_{k}\left(t_{j}\right) .
$$

Now, since $\beta_{k}\left(t_{j}\right) \rightarrow 0$ as $j \rightarrow \infty$, letting $j \rightarrow \infty$ in (52), we establish that $v(t)$ is a solution of $(20)$.

COROLlaRY 2. If $A(t) \geqq 0$ and there exists a function $B(t)$ such that

$$
\int_{t}^{\infty}(A(s)+B(s))^{2} d s \leqq B(t),
$$

then (1) is nonoscillatory.

We note that Corollary 2 reduces to Opial's condition (16) by taking $B(t) \equiv A(t)$. In view of the Wintner's Lemma, we can present independently a short proof of Corollary 2, hence the result (16) of Opial. 
Proof. Define $\varphi(t)=A(t)+\int_{t}^{\infty}(A(s)+B(s))^{2} d s \geqq 0$. A simple computation shows that $\varphi^{\prime}(t)+a(t)+(A(t)+B(t))^{2}=0$. Condition (53) implies that $\varphi^{2}(t) \leqq(A(t)+B(t))^{2}$, from which (19) readily follows. Hence (1) is nonoscillatory.

EXAMPLE 3. Consider the example $A(t)=(1+\sin t) / k t$. It is easily checked that in this case condition (16) fails for all $k>0$. On the other hand by taking $B(t)=1 / k t$, one can show that for large $t$, condition (53) is satisfied with $k>4 \frac{1}{2}$.

Example 4. Consider the example $A(t)=-(1+\sin t) / k t, k>0$ for which neither Opial's condition (16) nor Corollary 2 is applicable. However, in this case we may take $B(t)=4 / k t$, and easily check that condition (47) is satisfied for $k>5$.

In view of the other Riccati integral equations developed in the previous section, we can now apply similar techniques to (21) and (38) and obtain nonoscillation theorems. Note that in case of (21) and (38), $\bar{A}(t)$ and $\tilde{A}(t)$ are nonnegative, hence analogues of Theorem 4 take the same form as that of Corollary 2.

THEOREM 5. If there exists a function $\bar{B}(t)$ such that

$$
\int_{t}^{\infty}(\bar{A}(s)+\bar{B}(s))^{2} Q_{A}(s, t) d s \leqq \bar{B}(t)
$$

then (1) is nonoscillatory.

Proof. By Theorem 1, it is sufficient to show that (54) implies the existence of a solution of equation (21). Define $u_{0}(t)=A(t)+B(t)$ and inductively, $n=1,2,3, \ldots$

$$
u_{n}(t)=A(t)+\int_{t}^{\infty} Q_{A}(s, t) u_{n-1}^{2}(s) d s .
$$

Using (54) and (55), it is easy to show by induction that

$$
0 \leqq \bar{A}(t) \leqq u_{n}(t) \leqq u_{n-1}(t) \leqq \bar{A}(t)+B(t) .
$$

Thus the sequence of functions $\left\{u_{n}(t)\right\}$ has a pointwise limiting function $\tilde{u}(t)=$ $\lim _{n \rightarrow \infty} u_{n}(t)$. Since the integrand in (55) is nonnegative, it follows from the monotone convergence theorem that $\tilde{u}(t)$ is a solution of $(21)$.

Applying the same line of argument given in the proof of Theorem 5 to equation (38), we obtain

THEOREM 6. If $A(t)$ is integrable, and there exists a function $\widetilde{B}(t)$ such that

$$
\int_{t}^{\infty}(\tilde{A}(s)+\widetilde{B}(s))^{2} Q_{\bar{A}}(s, t) d s \leqq \tilde{B}(t)
$$

then (1) is nonoscillatory.

Taking $\bar{B}(t) \equiv \bar{A}(t)$ and $\widetilde{B}(t) \equiv \widetilde{A}(t)$ in the above two theorems, we obtain the following

Corollary 3. (i) (Willett [25]). If

$$
\int_{i}^{\infty} \bar{A}^{2}(s) Q_{A}(s, t) d s \leqq \frac{1}{4} \bar{A}(t)
$$


then (1) is nonoscillatory; and (ii) if $A(t)$ is integrable and

$$
\int_{t}^{\infty} \tilde{A}^{2}(s) Q_{A}(s, t) d s \leqq \frac{1}{4} \tilde{A}(t)
$$

then (1) is nonoscillatory.

We also note that our proof differs slightly from that of Willett [25] and both proofs are much simpler than that of Opial's [16]. We shall now present a further generalization of the oscillation part of Opial's result (17).

THEOREM 7. If $A(t) \geqq 0$ and there exists a nonnegative function $C(t) \not \equiv 0$ such that

$$
\int_{t}^{\infty} A^{2}(s) d s \geqq \frac{1}{4} A(t)+C(t),
$$

and

$$
\int_{t}^{\infty} C(s) A(s) d s \geqq \delta C(t),
$$

for some $\delta \geqq \frac{1}{4}$, then (1) is oscillatory.

Proof. Assume that (1) is nonoscillatory, then by Wintner's Lemma, equation (20) must have a solution $v(t)$, which satisfies $v(t) \geqq a_{0} A(t) \geqq 0$, with $a_{0}=1$. Substituting this into (20), we find

$$
v(t) \geqq a_{1} A(t)+b_{1} C(t), \quad a_{1}=1+\frac{1}{4}, b_{1}=1 .
$$

Substituting (61) again into (20), we obtain

$$
\begin{aligned}
v(t) & \geqq\left(1+a_{1}^{2} / 4\right) A(t)+2 a_{1} b_{1} \int_{t}^{\infty} C(s) A(s) d s+a_{1}^{2} C(t) \\
& =a_{2} A(t)+b_{2} C(t), \quad a_{2}=1+a_{1}^{2} / 4, b_{2}=a_{1}^{2}+2 \delta a_{1} b_{1} .
\end{aligned}
$$

Using (62) and an easy induction, we can show in general that

$$
v(t) \geqq a_{n} A(t)+b_{n} C(t), \quad a_{n}=1+a_{n-1}^{2} / 4, b_{n}=a_{n-1}^{2}+2 \delta a_{n-1} b_{n-1} .
$$

From the recurrence relation given by (63) and the fact that $a_{2} \geqq a_{1}, b_{2} \geqq b_{1}$, one readily sees that $a_{n} \geqq a_{n-1}, b_{n} \geqq b_{n-1}$. Furthermore, it is easy to show that $\lim _{n \rightarrow \infty} a_{n}$ $=2$. Now, if $\lim _{n \rightarrow \infty} b_{n}$ is finite, then we can show from (63) that $4 \delta<1$, contrary to the given hypothesis. Since $C(t) \not \equiv 0$, and $b_{n} \rightarrow \infty$ as $n \rightarrow \infty$, the desired contradiction follows from (63).

We observe that Opial's condition (17) follows from Theorem 7 by taking $C(t) \equiv(\varepsilon / 4) A(t)$ for some $\varepsilon>0$. Applying the same argument to equations (21) and (38), we obtain respectively:

THEOREM 8. If there exists a nonnegative function $\bar{C}(t) \not \equiv 0$ such that

$$
\int_{t}^{\infty} \bar{A}^{2}(s) Q_{A}(s, t) d s \geqq \frac{1}{4} \bar{A}(t)+\bar{C}(t),
$$


and

$$
\int_{t}^{\infty} \bar{C}(s) \bar{A}(s) Q_{A}(s, t) d s \geqq \delta \bar{C}(t),
$$

for some $\delta \geqq \frac{1}{4}$, then (1) is oscillatory.

THEOREM 9. If $A(t)$ is integrable and there exists a nonnegative function $\widetilde{C}(t) \not \equiv 0$ such that

$$
\int_{t}^{\infty} \tilde{A}^{2}(s) Q_{A}(s, t) d s \geqq \frac{1}{4} \tilde{A}(t)+\widetilde{C}(t),
$$

and

$$
\int_{t}^{\infty} \tilde{C}(s) \tilde{A}(s) Q_{\bar{A}}(s, t) d s \geqq \delta \tilde{C}(t),
$$

for some $\delta \geqq \frac{1}{4}$, then (1) is oscillatory.

Corollary 4. (i) (Willett [25]). If $\bar{A}(t) \not \equiv 0$ satisfies

$$
\int_{t}^{\infty} \bar{A}^{2}(s) Q_{A}(s, t) d s \geqq \frac{1+\varepsilon}{4} \bar{A}(t)
$$

for some $\varepsilon>0$, then (1) is oscillatory, (ii) if $\tilde{A}(t) \not \equiv 0$ satisfies

$$
\int_{t}^{\infty} \tilde{A}^{2}(s) Q_{\bar{A}}(s, t) d s \geqq \frac{1+\varepsilon}{4} \tilde{A}(t)
$$

for some $\varepsilon>0$, then (1) is oscillatory.

4. Comparison theorems. Consider another linear second order equation of the same form as that of (1)

$$
y^{\prime \prime}+b(t) y=0,
$$

we wish to discuss conditions on $a(t)$ and $b(t)$ such that nonoscillation of (1) would imply nonoscillation of (70), or vice versa. Indeed, Sturm's Comparison Theorem states the following: Suppose that for all large $t$,

$$
a(t) \geqq b(t),
$$

then the oscillation of (70) implies that (1) is oscillatory. In case both $a(t)$ and $b(t)$ are integrable, hence $A(t)$ and $B(t)=\int_{t}^{\infty} b(s) d s$ exist, Hille [8] showed that in this case if

$$
a(t), b(t) \geqq 0, \quad \text { and } \quad A(t) \geqq B(t),
$$

then, the same conclusion results. Hille's result was later improved by Wintner [24], who showed that condition (72) may be weakened to

$$
A(t) \geqq B(t) \geqq 0 .
$$


Later, Hartman [7] (p. 369, Exercise 7.9) indicated that Wintner's condition (73) may be improved to read

$$
A(t) \geqq|B(t)| .
$$

Hartman's result is particularly important in our discussion here, for it seems to be the first result of comparison type which is applicable when $B(t)$ is not necessarily nonnegative. In particular, we have

EXAMPLE 5. Consider $b(t)=(\alpha \sin \beta t) / t^{\gamma}$. Take $a(t)=1 / 4 t^{2}$ in (74), we conclude from the result of Hartman that (70) is nonoscillatory for all $\alpha, \beta$ if $\gamma>1$ and $|\alpha| \beta \mid<\frac{1}{4}$ if $\gamma=1$. On the other hand, Hartman's result gives no information concerning the oscillation of (1) when $A(t)$ assumes negative values of large $t$. In view of the Riccati integral equations, (21) and (38), introduced here, we can offer similar comparison theorems complementing that of Hartman's.

THEOREM 10. Suppose that $A(t)$ is integrable, and in addition we have

$$
\bar{A}(t) \geqq \bar{B}(t) \text { and } A(t)+\bar{A}(t) \geqq B(t)+\bar{B}(t),
$$

then if (1) is nonoscillatory, (70) is also nonoscillatory.

Proof. Suppose that (1) is nonoscillatory. By Theorem 1, equation (21) has a solution $u(t)$. Define $\rho(t)=\int_{t}^{\infty} u^{2}(s) Q_{A}(s, t) d s$, which satisfies:

$$
\rho^{\prime}(t)+\rho^{2}(t)+2(A(t)+\bar{A}(t)) \rho(t)+\bar{A}^{2}(t)=0 .
$$

Now define $z(t)=\exp \left(\int^{t} \rho(s) d s\right)$, which on account of (76) is a solution of the following second order equation:

$$
z^{\prime \prime}(t)+2(A(t)+\bar{A}(t)) z^{\prime}(t)+\bar{A}^{2}(t) z(t)=0 .
$$

Consider the second order equation with coefficients $B(t)$ and $\bar{B}(t)$ in the same form as (77)

$$
r^{\prime \prime}(t)+2(B(t)+\bar{B}(t)) r^{\prime}(t)+\bar{B}^{2}(t) r(t)=0 .
$$

Since $A(t)$ is integrable, nonoscillation of (1) implies on account of Theorem 2 that

$$
\int_{0}^{\infty} \exp \left(-2 \int_{0}^{t} A(u) d u\right) d t=\infty
$$

Note that $z(t)$ is nonoscillatory, this together with (79) shows that (77) has a solution $z(t)$ satisfying $z(t)>0, z^{\prime}(t) \geqq 0$. (Cf. Hartman [7], p. 352, Exercise 6.2.) Using this fact concerning equation (77) and (75), we may apply another result of Hartman ([7], p. 363, Exercise 7.2) to conclude that equation (78) is nonoscillatory which in turn implies that there is a solution to the following Riccati differential equation:

$$
\sigma^{\prime}(t)+\sigma^{2}(t)+2(B(t)+\bar{B}(t)) \sigma(t)+\bar{B}^{2}(t)=0 .
$$

Define $y(t)=\exp \left(\int_{0}^{t}(B(s)+\bar{B}(s)+\sigma(s)) d s\right)$. An easy computation shows that $y(t)$ is a nonoscillatory solution of (70). The proof is complete. 
EXAMPLE 6. Consider $a(t)=(\alpha \cos \beta t) / t^{\gamma}, 0<\gamma<1$. Take $b(t)=(\sin t) / t^{\lambda}$ for some $\lambda, \gamma<\lambda<1$. It is easily verified in this case that $A(t)$ is integrable and condition (75) is satisfied. Since equation (70) is oscillatory with this particular $b(t)$ from Example 2 , we conclude that (1) is oscillatory.

We remark that (46) is also applicable to Example 6. However, Theorem 10 offers comparison between coefficients $a(t), b(t)$, whose iterated integrals $A(t)$, $B(t)$ may both be negative for large $t$.

5. More examples. We shall now consider more elaborate examples demonstrating various applications of the results established in the previous two sections.

EXAMPLE 7. Consider $a(t)=(\alpha \sin \beta t) / t$. Using Corollary 4(i), it is easy to show that (1) is oscillatory if $|\alpha / \beta|>1 / 2^{1 / 2}$ and similarly using Corollary 3(i), we obtain nonoscillation of (1) when $|\alpha / \beta|<1 / 2^{1 / 2}$. (Details of these may be found in Willett [25].) We shall now show that Theorem 5 implies nonoscillation of (1) when $|\alpha / \beta|=1 / 2^{1 / 2}$. Note that in this case, we have

$$
\begin{aligned}
A(t) & =\frac{\alpha}{\beta} \frac{\cos \beta t}{t}+O\left(\frac{1}{t^{2}}\right), \\
Q_{A}(s, t) & =1+O(1 / t) \leqq 1+L / t, \quad L>0 .
\end{aligned}
$$

From (81) and (82), we obtain the following estimate for $\bar{A}(t)$ :

$$
\bar{A}(t)=\frac{\alpha^{2}}{2 \beta^{2}} \frac{1}{t}+O\left(\frac{1}{t^{2}}\right)
$$

Let $K>0$ be the constant such that $\bar{A}(t) \leqq 1 / 4 t+K / t^{2}$. We choose $\bar{B}(t)=1 / 4 t+M / t^{2}$, where $M$ is to be determined in terms of $K$ and $L$. Using (82),(83) and the estimates on $A$ and $\bar{A}$, we can compute the integral in (54) and obtain

$$
\int_{t}^{\infty}(A(s)+B(s))^{2} Q_{A}(s, t) d s=\frac{1}{4 t}+\frac{K+M+L / 4}{2 t^{2}}+O\left(\frac{1}{t^{3}}\right)
$$

In order that (54) be satisfied, it is sufficient to require: $M>K+L / 4$.

EXAMPLE 8. Consider $a(t)=(\alpha \sin \beta t) / t+\lambda / t^{2}$, with $\alpha, \beta, \lambda \neq 0$. Willett [25] shows that (1) is oscillatory if $\lambda>\frac{1}{4}-\frac{1}{2}(\alpha / \beta)^{2}$ and nonoscillatory if $\lambda<\frac{1}{4}-\frac{1}{2}(\alpha / \beta)^{2}$. Following a similar argument as outlined in Example 7, we find that (1) is nonoscillatory if $\lambda=\frac{1}{4}-\frac{1}{2}(\alpha / \beta)^{2}$. We note that in this case $A(t)$ is not integrable and estimate (82) for $Q_{A}(s, t)$ does not hold.

EXAMPLE 9. Consider $a(t)=(\alpha \sin \beta t) / t+(\mu \cos \nu t) / t$, with $\alpha, \beta, \mu, \nu \neq 0$. Applying Theorems 5 and 8 as before, we find that (1) is oscillatory if $(\alpha / \beta)^{2}+(\mu / \nu)^{2}>\frac{1}{4}$ and nonoscillatory if $(\alpha / \beta)^{2}+(\mu / \nu)^{2} \leqq \frac{1}{4}$.

EXAMPLE 10. Consider $a(t)=(\alpha \sin \beta t) / t+(\mu \sin v t) / t^{\sigma}$, with $|\alpha / \beta|=1 / 2^{1 / 2}, \sigma>0$, $\nu \neq \beta$. In this case, we find that (1) is oscillatory if $\sigma \leqq 1$ and nonoscillatory if $\sigma>1$.

Similar to the role that equation (18) plays with regard to equation (4), we also consider the logarithmetic perturbation to the boundary case of Example 7, i.e. $|\alpha / \beta|=1 / 2^{1 / 2}$. 
ExAmple 11. Consider $a(t)=(\alpha \sin \beta t) / t+(\xi \sin \eta t) / t \log t$, with $|\alpha / \beta|=1 / 2^{1 / 2}$, $\xi, \eta \neq 0$.

Case I, $\beta=\eta$, we have

$$
\begin{aligned}
& A(t)=\frac{\alpha \cos \beta t}{\beta t}+\frac{\xi \cos \beta t}{\beta t \log t}+O\left(\frac{1}{t^{2}}\right) \\
& \bar{A}(t)=\frac{1}{4 t}+\frac{2 \alpha \xi}{\beta^{2} t \log t}+O\left(\frac{1}{t(\log t)^{2}}\right)
\end{aligned}
$$

and

$$
\int_{t}^{\infty} \bar{A}^{2}(s) Q_{A}(s, t) d s=\frac{1}{16 t}+\frac{\alpha \xi}{\beta^{2} t \log t}+O\left(\frac{1}{t(\log t)^{2}}\right) .
$$

Substituting (85) and (86) in (59) and (60), and applying Theorem 8, we conclude that (1) is oscillatory if $\alpha \xi>0$, i.e. $\xi$ has the same sign as $\alpha$. As a direct consequence of Theorem 5, we also have that (1) is nonoscillatory provided that $\alpha \xi<0$. In this example, (84) implies that $A(t)$ is integrable, so Theorems 6 and 9 are also applicable and indeed they produce the same conclusion. Note that Willett's results [25] in particular are not applicable here, and hence give no information for this case.

Case II, $\beta \neq \eta$, we have

$$
\bar{A}(t)=\frac{1}{4 t}+\frac{1}{2}\left(\frac{\xi}{\eta}\right)^{2} \frac{1}{t(\log t)^{2}}+O\left(\frac{1}{t^{2}}\right)
$$

and

$$
\int_{t}^{\infty} \bar{A}^{2}(s) Q_{A}(s, t) d s=\frac{1}{16}+\frac{1}{4}\left(\frac{\xi}{\eta}\right)^{2} \frac{1}{t(\log t)^{2}}+O\left(\frac{1}{t(\log t)^{3}}\right)
$$

Using (87) and (88) and a direction application of Theorem 8, we conclude that (1) is oscillatory in this case.

EXAMPLE 12. Consider $a(t)=\mu \sin v t^{2}$. Since $a(t)$ is integrable, our results are applicable. It can easily be computed that in this case

$$
A(t)=\frac{\mu}{2 v} \frac{\cos v t^{2}}{t}+O\left(\frac{1}{t^{2}}\right)
$$

and

$$
\bar{A}(t)=\frac{1}{2}\left(\frac{\mu}{2 v}\right)^{2} \frac{1}{t}+O\left(\frac{1}{t^{2}}\right) .
$$

Applying similar techniques as before, we obtain oscillation for $|\mu / \nu|>1$ and nonoscillation for $|\mu / \nu| \leqq 1$.

We remark that in all examples discussed above the conclusions remain valid if sines and cosines are interchanged or the coefficient $A(t)$ has a perturbation term of order $O\left(1 / t^{\lambda}\right), \lambda>1$. Also note that part of the results may be obtained by a combination of Theorem 10 and Theorems 5 through 9 . It is believed that in case $A(t)$ is integrable, Theorems 6 and 9 will be useful in discussing perturbations of higher order, namely $O\left((t \log t \log |\log t|)^{-1}\right)$. 
6. Extensions and remarks. Using transformation of variables, one can easily translate results concerning equation (1) to the general Sturm-Liouville equation of second order:

$$
\left(p(t) x^{\prime}\right)^{\prime}+q(t) x=0, \quad p(t)>0 .
$$

Following Moore [13], we can transform equation (89) into the same form as equation (1), depending on the convergence or divergence of the integral $\int^{\infty} d t / p(t)$. In the first case, we introduce the change of variables

$$
s=\int_{0}^{t} \frac{d \tau}{p(\tau)}, \quad y(s)=x(t)
$$

which transforms (89) into

$$
\frac{d^{2} y}{d s^{2}}+p(t) q(t) y(s)=0, \quad s \in\left[s_{0}, \infty\right) .
$$

In the other case, we introduce the transformation

$$
s=\left(\int_{t}^{\infty} \frac{d \tau}{p(\tau)}\right)^{-1}, \quad s^{-1} y(s)=x(t)
$$

which transforms (91) into

$$
\frac{d^{2} y}{d s^{2}}+\frac{p(t) q(t)}{s^{4}} y(s)=0, \quad s \in\left[s_{0}, \infty\right) .
$$

In both cases, equations (92) and (93) are of the same form of (1) to which results based upon (1) are readily applicable. Some results on asymptotic behavior of equation (89) and in particular, oscillation or nonoscillation results, which have appeared in literature are for the most part simple consequences of transformations (90), (92), or some slightly more general transformations as given in [13]. This phenomenon occurs also in its nonlinear analogue, Willett [25] and Wong [27] listed a few references of this nature.

In order that results in this paper be applicable to equation (89), we require instead of condition (13) the equivalent conditions on $p(t)$ and $q(t)$ :

$$
\int^{\infty} \frac{d t}{p(t)}=\infty, \quad \int^{\infty} q(t) d t<\infty
$$

or

$$
\int^{\infty} \frac{d t}{p(t)}<\infty, \quad \int^{\infty} q(t)\left(\int_{t}^{\infty} \frac{d \tau}{p(\tau)}\right)^{2} d t<\infty
$$

Under assumptions (94) and (95), we can introduce functions $Q_{p}(t)$, and $\bar{Q}_{p}(t)$ for equation (89) which play the same role as that of $A(t)$ and $\bar{A}(t)$ for equation (1). 
In case condition (94) holds, an easy computation yields the following formulae for $Q_{p}$ and $\bar{Q}_{p}$ :

$$
\begin{aligned}
& Q_{p}(t)=\int_{t}^{\infty} q(\tau) d \tau \\
& \bar{Q}_{p}(t)=\int_{t}^{\infty} \frac{Q_{p}^{2}(\tau)}{p(\tau)} \exp \left(2 \int_{t}^{\tau} \frac{1}{p(\xi)} Q_{p}(\xi) d \xi\right) d \tau .
\end{aligned}
$$

Similarly, when condition (95) is satisfied, we have the alternative formulae for $Q_{p}$ and $\bar{Q}_{p}$.

$$
\begin{aligned}
& Q_{p}(t)=\int_{t}^{\infty} q(\tau)\left(\int_{\tau}^{\infty} \frac{d \xi}{p(\xi)}\right)^{2} d \tau \\
& Q_{p}(t)=\int_{t}^{\infty} \frac{Q_{p}^{2}(\tau)}{p(\tau)} \exp \left(2 \int_{t}^{\tau} \frac{Q_{p}(\xi)}{p(\xi)}\left(\int_{\xi}^{\infty} \frac{d \eta}{p(\eta)}\right)^{-2} d \xi\right)\left(\int_{\tau}^{\infty} \frac{d \xi}{p(\xi)}\right)^{-2} d \tau
\end{aligned}
$$

It is rather important to point out that transformations (90) and (92) are just two of many possible "oscillation invariant" transformations, i.e. $s \rightarrow \infty$ as $t \rightarrow \infty$ and $y(s)$ vanishes whenever $x(t)$ does. In many instances when oscillation or nonoscillation criteria fail to apply to equation (1) directly, some appropriate oscillation invariant transformation may be introduced so that in the reduced equation (89) results on oscillation or nonoscillation may be applicable. In this regard, we discuss the following two examples.

EXAMPLE 13. Consider the following equation with independent variable $s$ :

$$
\frac{d^{2} y}{d s^{2}}+(\cos s) y=0, \quad s \geqq 0 .
$$

Since $\cos s$ is not integrable on $[0, \infty)$, our results are not directly applicable to equation (96). However, by introducing the "oscillation invariant" transformation:

$$
s=\log t, \quad y(s)=t x(t),
$$

we may transform equation (96) into the form (89) as follows:

$$
\left(t^{3} x^{\prime}\right)^{\prime}+(t+t \cos \log t) x=0,
$$

to which our results are applicable. In particular, an easy application of Corollary 1(i) to equation (97) with the above-mentioned modification, replacing $Q_{p}, \bar{Q}_{p}$ for $A$ and $\bar{A}$, yields oscillation of all solutions of (96).

EXAMPLE 14. Consider the following equation with independent variable $t$ :

$$
x^{\prime \prime}+\left(\mu t \cos \nu t^{2}\right) x=0, \quad \mu, \nu \neq 0 .
$$

Obviously, none of the above-mentioned results are applicable here. Now, using the following "oscillation invariant" transformation:

$$
2 s=t^{2}, \quad y(s)=t x(t),
$$


we can transform (98) into:

$$
\frac{d}{d s}\left(s^{-1} y^{\prime}(s)\right)+\left(\frac{2}{s^{3}}+\frac{\mu \cos \nu s}{s^{3 / 2}}\right) y(s)=0 .
$$

Since (99) is of the form (89), an easy verification of (94) shows that our results are applicable here. In fact, using any oscillation criteria above, we obtain the conclusion that (98) is oscillatory for all $\mu, \nu \neq 0$.

Finally, we close our discussion with a number of remarks relating our present work to others. (Other related results may be found in Reid [19], [20].)

REMARK 1. For nonnegative coefficients $a(t)$ other results are known. See for example, Nehari [15], Barrett [1]. We note that our results are best possible in the sense that in cases Euler's equation (4) and the Riemann-Weber equation (18) inequalities concerning $A(t), \bar{A}(t)$ become equalities.

REMARK 2 . Examples 13 and 14 belong to a class of so-called "wildly oscillating" coefficients. For these examples, the technique of averaging plays an important role. (Cf. Wintner [22], Hartman [6], Coles [2], Willett [25], Macki and Wong [11], and Coles and Willett [4].) The fact that some of these cases can be handled by some appropriate oscillation invariant transformations via results on integrable coefficient seems not yet fully understood. On the other hand, Macki and Wong [11] demonstrate an example with integrable coefficient which can be handled by averaging methods.

REMARK 3. The behavior of nonoscillatory solutions has been studied by a number of authors, under the assumption that $a(t)$ must eventually be of one sign (see Levin [10], and other references listed there). In that case, specific information on the limiting behavior of nonoscillatory solutions are known. In view of the present development it will be of interest to improve the above-mentioned results by relaxing the sign restriction on $a(t)$.

REMARK 4. Marcus and Moore [12] have presented a detailed discussion of disconjugacy domains of solutions of (1) when $a(t)$ is nonnegative and almost periodic. We hope that the present development will lead to an improvement of their results when $a(t)$ is not required to be nonnegative.

\section{REFERENCES}

1. J. H. Barrett, Disconjugacy of second order linear differential equations with nonnegative coefficients, Proc. Amer. Math. Soc. 10 (1959), 552-561.

2. W. J. Coles, An oscillation criterion for second order linear differential equations, Proc Amer. Math. Soc. 19 (1968), 755-759.

3. - A simple proof of a well-known oscillation theorem, Proc. Amer. Math. Soc. 19 (1968), 507.

4. W. J. Coles and D. Willett, Summability criteria for oscillation of second order linear differential equations, Ann. Mat. Pura Appl. (4) 79 (1968), 391-398.

5. W. B. Fite, Concerning the zeros of the solutions of certain differential equations, Trans. Amer. Math. Soc. 19 (1918), 341-352. 
6. P. Hartman, On nonoscillatory linear differential equations of second order, Amer. J. Math. 74 (1952), 389-400.

7. - Ordinary differential equations, Wiley, New York, 1964.

8. E. Hille, Nonoscillation theorems, Trans. Amer. Math. Soc. 64 (1948), 234-252.

9. A. Kneser, Untersuchungen über die reellen Nullstellen der Integrale linearer Differentialgleichungen, Math. Ann. 42 (1893), 409-435.

10. A. Ju. Levin, Classification of nonoscillatory cases for the equation $x^{\prime \prime}+p(t) x^{\prime}+q(t) x=0$ where $q(t)$ is of constant sign, Dokl. Akad. Nauk SSSR 171 (1966), 1037-1040=Soviet Math. Dokl. 7 (1966), 1599-1602.

11. J. W. Macki and J. S. W. Wong, Oscillation theorems for linear second order differential equations, Proc. Amer. Math. Soc. 20 (1969), 67-72.

12. L. Marcus and R. A. Moore, Oscillation and disconjugacy for linear differential equations with almost periodic coefficients, Acta Math. 96 (1956), 99-123.

13. R. A. Moore, The behavior of solutions of a linear differential equation of second order, Pacific J. Math. 5 (1955), 125-145.

14. - The least eigenvalue of Hill's equation, J. Analyse Math. 5 (1956/1957), 183-196.

15. Z. Nehari, Oscillation criteria for second order linear differential equations, Trans. Amer. Math. Soc. 85 (1957), 428-445.

16. Z. Opial, Sur les intégrales oscillantes de l'equation differentielle $u^{\prime \prime}+f(t) u=0^{\prime}$, Ann. Polon. Math. 4 (1958), 308-313.

17. - Sur une critere d'oscillation des integrales de l'equation differentielle $\left(Q(t) x^{\prime}\right)^{\prime}$ $+f(t) x=0$, Ann. Polon. Math. 6 (1959-1960), 99-104.

18. B. Riemann and H. Weber, Die Partiellen Differentialgleichungen der Mathematischen Physik. II, 5th ed., Brunschweig, 1912.

19. W. T. Reid, Oscillation criteria for linear differential systems with complex coefficients, Pacific J. Math. 6 (1956), 733-751.

20. - Oscillation criteria for self-adjoint differential systems, Trans. Amer. Math. Soc. 101 (1961), 91-106.

21. C. Sturm, Sur les equations differentielles lineaires du second ordre, J. Math. Pures Appl. 1 (1836), 106-186.

22. A. Wintner, A criteria of oscillatory stability, Quart. Appl. Math. 7 (1949), 115-117.

23. - On the nonexistence of conjugate points, Amer. J. Math. 73 (1951), 368-380.

24. - On the comparison theorem of Kneser-Hille, Math. Scand. 5 (1957), 255-260.

25. D. Willett, On the oscillatory behavior of the solutions of second order linear differential equations, Ann. Polon. Math. (to appear).

26. J. S. W. Wong, Second order linear oscillation with integrable coefficients, Bull. Amer. Math. Soc. 74 (1968), 909-911.

27. - On second order nonlinear oscillation, Math. Research Ctr., Univ. of Wisconsin, Technical Report No. 836, also Funkcial. Ekvac. 11 (1969), 207-234.

Carnegie-Mellon University, Pittsburgh, Pennsylvania 